\title{
ON ILLUMINATION EFFECT IN AGN DISKS
}

\author{
INSU YI \\ Harvard-Smithsonian Center for Astrophysics \\ 60 Garden St., Cambridge, MA 02138, USA
}

We consider illumination effects in a weakly self-gravitating $\left(4 \pi G \Sigma \sim \Omega^{2} z\right)$ outer disk region $\left(R \sim 10^{17} \mathrm{~cm}\right)$ around a supermassive blackhole of $M_{8}=$ $M / 10^{8} M_{\text {sun }}=1$ with relatively high mass accretion rates $\left(\dot{M}_{25}=\dot{M} / 10^{25} \mathrm{~g} / \mathrm{s}=\right.$ 1). (e.g., Shlosman and Begelman 1989, APJ, 341, 685; Shore and White 1982, APJ, 256, 390).

We adopt an effective gravitational viscosity which is characterized by $\nu_{\text {eff }} \sim$ $\left(G / M^{3}\right)^{1 / 2} \Sigma^{2} R^{9 / 2}$ (Lin and Pringle 1987, MNRAS, 225, 607). The illumination is dominant over the local viscous dissipation for $R>3 G M / 2 c^{2} \epsilon \eta=2.2 \times$ $10^{15} M_{8}(\epsilon \eta)_{0.01}^{-1} \mathrm{~cm}(\epsilon$ is the fraction of energy emitted by the central hard X-ray source, $\eta$ is the efficiency of accretion). We consider the region where $P=P_{g a s} \gg$ $P_{\text {radiation }}$ and $\tau_{f f} \gg \tau_{e s}$. We calculate the vertical structure and self-consistently determine the disk parameters assuming that the illuminating photon energies are of a single value, $E=10 \mathrm{keV}$. We find $H \propto R^{0.6}$ and $T_{c} \propto R^{-0.8}$ (cf. for a standard $\alpha$ disk $H \propto R^{8 / 9}$ and $\left.T_{c} \propto R^{-3 / 4}\right)$. For a fixed set of model parameters $(\dot{M}, M$, $R$, and $E)$, we vary the fraction $(\epsilon \eta)$ of accretion energy, which is converted into illumination flux, from 0.001 to 0.1 . We calculate expected model spectra from a radial zone $R=10^{17}-3 \times 10^{17} \mathrm{~cm}$. When the illuminating photon energy is fixed, as the fraction increases from 0.001 to 0.1 , the peak photon energy increases from $10^{3} \mathrm{~K}$ to $3.2 \times 10^{3} \mathrm{~K}$. Although the hard X-ray photons do not penetrate the AGN disks into the central region, the surface heating and resulting redistribution of energy affects the disk structure and emission. For a fixed set of accretion parameters, with $\epsilon \eta=0.01$, we vary the characteristic energy $E$ of the illuminating hard-X-ray flux. Due to energy dependence of X-ray absorption depth, the vertical structure is significantly affected by varying incident photon energies even when the illumination flux is kept constant.

The results described above are interestingly related to the following analytically derived radial structure of accretion disks for the same region, $T_{c} \approx[3.7 \times$ $\left.10^{5} \mathrm{~K}\right] M_{8}^{3 / 17} \dot{M}_{25}^{4 / 17} R_{15}^{-13 / 17}(\epsilon \eta)_{0.01}^{2 / 17}, \Sigma \approx\left[2.3 \times 10^{7} \mathrm{~g} / \mathrm{cm}^{2}\right] M_{8}^{1 / 2} \dot{M}_{25}^{1 / 3} R_{15}^{-3 / 2}$, and $H \approx\left[5.3 \times 10^{12} \mathrm{~cm}\right] M_{8}^{-11 / 34} \dot{M}_{25}^{-5 / 51} R_{15}^{25 / 34}(\epsilon \eta)_{0.01}^{2 / 17}$. The results of numerical calculations are roughly in qualitative agreement with the above analytic results.

T. J.-L. Courvoisier and A. Blecha: Multi-Wavelength Continuum Emission of AGN, 493. (C) 1994 IAU. Printed in the Netherlands. 